\title{
Prognostic Value of PET/CT and ALDH1 in Pancreatic Cancer and Correlation of Standardized Uptake Values with Clinical Characteristics and Survival
}

\author{
DINA MOGHAZY MOHAMED, M.D.*; DAREEN ABDULAZIZ, M.D.** and \\ RANIA SOBHY ABOUKHADRAH, M.D.* \\ The Departments of Radiology* and Pathology**, Faculty of Medicine, Tanta University
}

\begin{abstract}
Background: Pancreatic malignancy is a rapidly growing cause of death in the world. Its survival rate is months even if discovered early.

Aim of Study: We evaluated the correlations between PET/SUVs (Standard Uptake Values), clinical characteristics and cancer stem-like cells named aldehyde dehydrogenase 1 (ALDH1) in pancreatic cancer. We evaluated the diagnostic performance of SUV and its correlation with overall survival.

Patients and Methods: Thirty-nine patients with pancreatic cancer were evaluated by PET/CT. The expression of ALDH1 was detected immunohistochemically in their cancer specimens. Results of PET/SUV were correlated with clinicpathological character, ALDH1 and overall survival.

Results: PET/SUV was associated significantly with tumor size ( $p=0.027)$. No significant correlation with other clinicalpathological characteristics. PET/CT showed sensitivity= $81 \%$, specificity $=78 \%$ and accuracy $=82 \%$. PET/SUV reflected the expression level of ALDH1 with significant correlation $(p=0.001)$. Patients with high SUV and high expression of ALDH1 had a worse median overall.

Conclusion: PET-CT was a non-invasive imaging modality that helped in pancreatic cancer especially in doubtful tumors. PET/CT was helpful tool to detect lymph nodes invasion and distant metastasis. It was associated with the expression level of ALDH1. High SUV and high expression of ALDH1were significantly correlated with a worse clinical outcome. PET/SUV and Cancer stem cells may serve as significant prognostic factors in predicting outcomes in pancreatic cancer patients.
\end{abstract}

Key Words: Pancreatic carcinoma - PET/SUV - ALDH1 Survival.

\section{Introduction}

PANCREATIC malignancy is a rapidly growing cause of death in the world. Its survival rate is

Correspondence to: Dr. Dina Moghazy Mohamed, The Department of Radiology, Faculty of Medicine, Tanta University months even if discovered early. The incidence of mortality rate is rapidly increasing even in developing countries due to many contributing factors including smoking, diet, pancreatitis, physical inactivity and genetic mutation [1].

Malignant pancreatic tumors include pancreatic excorine tumors mainly adenocarcinoma, neuroendocrine tumor, mucinous tumor and other rare forms of lymphoma [2].

Ultrasound has limited role in pancreatic imaging. Bowel gas handers the visualization of pancreas. Biliary tract dilatation secondly to pancreatic head cancer can be visualized by ultrasound but it will be difficult to visualize the tumor itself [3] .

Endoscopic retrograde cholangiopancreatography (ERCP) is a helpful test in evaluation the obstruction in either biliary or pancreatic levels. Placement of a stent in a biliary tree can help relieve symptoms of obstruction in some advanced patient [4]

Multi-detector computed tomography (MDCT) is the best imaging modality in patients with suspected pancreatic cancer. It helps in diagnosis and evaluation of local tumor invasion and distant spread [5].

Magnetic resonance imaging and magnetic resonance cholangiopancreatography (MRCP) with IV contrast is helpful in the preoperative assessment of pancreatic cancer and invasion of vascular structure [4].

Positron emission tomography (PET) is a new functional imaging modality used in detection of tumor. PET lacks the high spatial resolution for actual detection of the site of the tumor. Combined 
$\mathrm{PET} / \mathrm{CT}$ is now consider to have promising role in early diagnosis of pancreatic tumor as it carries the advantage of both anatomical and functional imaging of patient with suspected pancreatic mass

The 5-year survival rate for patients with pancreatic cancer has little changed in spite of introduction of new chemotherapy drugs. In attempt to increase patients' survival rate in recent years, researchers pay attention in identifying cancer stem cells (CSCs) CD133, CD24, CD44, EPCAM, ESA, c-Met, Aldh1, and more recently DclK1 and Lgr5

CSCs have two properties: Their ability to produce differentiated progeny that helps in recapitulating the original tumor structure and their capacity of long term proliferation of the malignant cell [8]. The cellular physiological processes that control the self-renewal of pancreatic malignancy are beginning to be described. They contain signaling tracts that show activity during early embryonic growth [9]. ALDH1 is a specific protein isoforms have been recognized as CSC markers in different type of cancer. It was found that there is a significant correlation between ALDH1 expression and poor overall survival for patients with pancreatic carcinoma [10]

Despite of the fact that CSCs have a central part in the beginning of disease, progress and recurrence, their state and clinical behavior have been the matter of debate. CSCs may be also show resistance to chemotherapy drugs [11]

In this study, we evaluated the correlations between standardized uptake values (SUVs) which assessed by positron emission tomography (PET) and clinical characteristics as well as cancer stemlike cells namely (ALDH1) in patients with cancer pancreas. In addition, we evaluated the diagnostic performance of SUV and its correlation with overall survival.

\section{Patients and Methods}

\section{Patients population:}

This retrospective study included 39 patients. Our study was approved by ethical committee of Tanta university hospital. All patients are informed about the study and gave written consent.

\section{This study included:}

1- Patients were suspected of having pancreatic cancer on the basis of MDCT.

2- Patients underwent PET/CT scan and pancreatic cancer stem cells namely (ALDH1).
3- Patients who underwent subsequent surgery or biopsy to confirm the histological type of the lesion.

\section{The exclusion criteria were:}

1- Those patients did not undergo surgical management with no available histological results.

2- Patients underwent chemotherapy/radiation prior to initial PET/CT scan.

\section{PET/CT scan:}

\section{Preparation:}

Included patients should be fasting at least 6 hours. Full clinical history about diabetes, random sugar (to assess the glucose level) and renal function tests are required. All metallic objects were removed and patients wore a specific gown. An intravenous cannula was inserted for 18F-FDG administration. The patients were asked to avoid any stressful condition to avoid physiological uptake of $18 \mathrm{~F}$ FDG by the active muscles and to void before examination.

Patients were instructed to drink about one and half liters of water which act as neutral oral contrast agent $1 \mathrm{~h}$ before the examination. Ten $\mathrm{mCi}(1 \mathrm{ml} /$ $10 \mathrm{~kg}$ ) of $18-\mathrm{F}-\mathrm{FDG}$ was injected manually $45-60$ min before starting the examination.

\section{Data extraction and processing:}

Fused PET/CT scans were performed using hybrid PET/CT system (Philips, NM Gemini, the Netherlands) which is consist of a dedicated PET scanner integrated with a 16 multi-slice CT scanner. The patients were positioned in a comfortable head fixation with arms up. CT scan was performed from vault to foot first. The following parameters $140 \mathrm{kV}, 60 \mathrm{~mA}, 5 \mathrm{~mm}$ slice thickness and $0.5 \mathrm{~mm}$ incrimination were used. The duration of the study was approximately $25-35 \mathrm{~min}$. The patients asked to breath quietly during the study. PET scan immediately followed the CT study without moving the patient. Helical PET and CT images were reconstructed then reformatted into coronal and sagittal images. PET and CT fusion images were generated by combining the 2 types of data. The sets of PET image data were reconstructed using CT data for attenuation correction and co-registered scan images were displayed using special software and reviewed on the workstation.

\section{Image analysis of PET/CT scan:}

Any abnormal focal FDG accumulation in the pancreas exceeding normal regional uptake was indicative of malignancy [12] 


\section{Immunohistochemistry:}

All pathologic slides were reanalyzed by an experienced pathologist. Tumor sections were fixed in formalin, embedded in paraffin, sectioned and stained with hematoxylin and eosin (H\&E). Immunohistochemistry (IHC) with the anti-ALDH1 antibody (Abcam) was performed using a Dako Envision Kit following the manufacturers' instructions. The immunoreaction was evaluated according to the estimated staining intensity according to the following parameter: $0=$ no staining, $+=$ weak staining, $++=$ moderate staining,$+++=$ strong staining, and $++++=$ very strong staining. 0 and + are considered negative (low level) while ++ , +++ and ++++ are considered positive (high level) [13].

\section{Follow-up:}

Follow up of our patients included regular physical examination and blood tests (liver, kidney functions and tumor markers). CT scan was not needed regularly depending on patient's symptoms. Follow-up with oncologist after surgery was every 3 to 6 months for 1 to 2 years, every 6 to 12 months for 3 to 5 years and then annually. Follow-up period ranged between 14 and 96 months with a median of 29 months.

\section{Statistical analysis:}

Fisher's exact test was used to assess the significant differences between the clinical characteristics of included patients and PET-SUV values. The overall survival (OS) was measured from the time of resection until death or last follow-up. Survival analysis for differences among the higher and lower SUV values and pancreatic cancer stem cells (ALDH1) was performed using the KaplanMeier method.

Using surgery and histo-pathological results as gold standard, the sensitivity, specificity and accuracy of the SUV in the diagnosis of pancreatic cancer were assessed. The analyses were performed using SAS version 20 (SAS Inc., Cary, NC).

\section{Results}

Demographic and clinical characteristics of included patients:

Demographic and clinical characteristics of the 39 patients with suspected pancreatic cancer by MDCT illustrated in Table (1). A male prominence was detected in this study represented $64 \%$ while female presented $36 \%$. Ages of patients ranged from 50-70 years with mean age about 60 .
Histopathological results revealed that pancreatic adenocarcinoma was diagnosed in $54 \%$ of patients, neuroendocrine and mucinous carcinomas were in the remaining patients.

Regarding histopathologicl differentiation,the tumor was moderately differentiated in $64 \%$ of patients, poorly differentiated in $15 \%$ of patients and well differentiated in the remaining patients.

The common site of the pancreatic masses in this study were in the head $(58 \%)$ followed by the body and tail in $42 \%$ of patients. Seventeen patients (43\%) showed evidence of lymph node invasion at the time of examination diagnosed in PET-CT scan.

Twenty-six patients (67\%) showed evidence of extra-pancreatic metastasis. Distant sites of metastasis included the liver $(\mathrm{N}=12)$, lung $(\mathrm{N}=7)$, and multiple organs ( $\mathrm{N}=7$ patients).

Regarding tumor staging system which confirmed by endoscopic biopsy, $66 \%$ of patients had clinical Stage III. The expression of ALDH1 in patients with cancer pancreas were high in $58 \%$ of patients and low in $42 \%$ of patients.

Table (1): Demographic and clinical characteristics of included patients $(\mathrm{N}=39)$.

\begin{tabular}{|c|c|c|c|}
\hline \multicolumn{2}{|l|}{ Demographic data } & \multirow{2}{*}{$\frac{N}{25}$} & \multirow{2}{*}{$\frac{\%}{64}$} \\
\hline Sex & Male & & \\
\hline & Female & 14 & 36 \\
\hline \multirow[t]{2}{*}{ Age } & $>50$ years & 26 & 68 \\
\hline & $<50$ years & 13 & 32 \\
\hline \multirow{3}{*}{$\begin{array}{l}\text { Histopathological } \\
\text { analysis }\end{array}$} & Adenocarcinoma & 21 & 54 \\
\hline & Neuroendcorine tumor & 9 & 23 \\
\hline & Mucinous carcinoma & 9 & 23 \\
\hline \multirow[t]{2}{*}{ Site of tumor } & Head & 23 & 58 \\
\hline & Body/tail & 16 & 42 \\
\hline \multirow[t]{3}{*}{ TNM Stage } & I & 6 & 16 \\
\hline & II & 7 & 18 \\
\hline & III & 26 & 66 \\
\hline \multirow{3}{*}{$\begin{array}{l}\text { Histological } \\
\text { differentiation }\end{array}$} & Well & 8 & 21 \\
\hline & Moderate & 25 & 64 \\
\hline & Poor & 6 & 15 \\
\hline \multirow[t]{2}{*}{$\mathrm{N}$ stage } & No & 22 & 56 \\
\hline & N 1 & 17 & 43 \\
\hline \multirow[t]{2}{*}{ M stage } & Present & 26 & 67 \\
\hline & Absent & 13 & 33 \\
\hline \multirow[t]{2}{*}{ ALDHIAI } & High & 23 & 58 \\
\hline & Low & 16 & 42 \\
\hline
\end{tabular}


Correlation of PET-SUV with patient's clinicopathologic characteristics and ALDHI:

The mean SUV by PET-CT of 4.5 was used as a cut-off value to detect the activity of the pancreatic mass. Out of 39 patients, 27 patients $(69 \%)$ showed high PET/SUV (more than 4.5) and 12 patients (31\%) had low PET/SUV (less than 4.5) (Table 2). There were no significant difference between patients with high and low PET/SUV lesions regarding age, gender and location of the tumor $(p=$ $0.661, p=0.515, p=0.784)$. Nineteen patients $(49 \%)$ with high expression of ALDH1 showed SUV / PET-CT more than 4.5. Eight patients (20\%) with low expression of ALDH1 showed PET/SUV more than 4.5. There was statically significant difference between both parameters $(p=0.001)$.In this study, the median tumor size was $3 \pm 1.01 \mathrm{~cm}$. PET/SUV $>4.5$ was found in tumor size more than $3 \mathrm{~cm}$ in 6 patients and less than $3 \mathrm{~cm}$ in 7 patients with $\mathrm{PET} / \mathrm{SUV} \leq 4.5$. Tumor size was found to have significant correlation with PET/SUV ( $p=0.027)$. No statistically significant correlation was found between malignant pancreatic mass with high or low PET/SUV neither with TNM staging system $(p=0.644)$ nor with histologic differentiation $(p=0.898)$.

Table (2): Correlation of tumor (PET-SUV) with patient's clinico-pathologic characteristics \& ALDH1.

\begin{tabular}{|c|c|c|c|c|c|}
\hline & & $\begin{array}{c}>4.5 \\
(\mathrm{n}=27)\end{array}$ & $\begin{array}{c}\leq 4.5 \\
(\mathrm{n}=12)\end{array}$ & $X^{2}$ & $\begin{array}{c}p- \\
\text { value }\end{array}$ \\
\hline Age & $\begin{array}{l}>50 \text { years } \\
\leq 50 \text { years }\end{array}$ & $\begin{array}{l}16 \\
11\end{array}$ & $\begin{array}{l}8 \\
4\end{array}$ & 0.193 & 0.661 \\
\hline Gender & $\begin{array}{l}\text { Male } \\
\text { Female }\end{array}$ & $\begin{array}{l}15 \\
12\end{array}$ & $\begin{array}{l}8 \\
4\end{array}$ & 0.423 & 0.515 \\
\hline ALDH1A1 & $\begin{array}{l}\text { High } \\
\text { Low }\end{array}$ & $\begin{array}{l}23 \\
4\end{array}$ & $\begin{array}{l}1 \\
11\end{array}$ & 18.340 & $0.001 *$ \\
\hline Tumor location & $\begin{array}{l}\text { Head } \\
\text { Body/tail }\end{array}$ & $\begin{array}{l}17 \\
10\end{array}$ & $\begin{array}{l}7 \\
5\end{array}$ & 0.083 & 0.784 \\
\hline $\begin{array}{l}\text { Tumor size }(\mathrm{cm}) \\
\text { Median } 3 \mathrm{~cm} \\
(1.0-11.1)\end{array}$ & $\begin{array}{l}>3 \\
\leq 3\end{array}$ & $\begin{array}{l}6 \\
21\end{array}$ & $\begin{array}{l}7 \\
5\end{array}$ & 4.882 & $0.027 *$ \\
\hline TNM Stage & $\begin{array}{l}\text { I } \\
\text { II / III }\end{array}$ & $\begin{array}{l}5 \\
22\end{array}$ & $\begin{array}{l}3 \\
9\end{array}$ & 0.213 & 0.644 \\
\hline $\begin{array}{l}\text { Histologic } \\
\text { differentiation }\end{array}$ & $\begin{array}{l}\text { Well } \\
\text { Moderate } \\
\text { Poor }\end{array}$ & $\begin{array}{l}5 \\
17 \\
5\end{array}$ & $\begin{array}{l}3 \\
7 \\
2\end{array}$ & 0.208 & 0.898 \\
\hline $\mathrm{N}$ stage & $\begin{array}{l}\text { N } 0 \\
\text { N } 1\end{array}$ & $\begin{array}{l}14 \\
13\end{array}$ & $\begin{array}{l}8 \\
4\end{array}$ & 0.741 & 0.389 \\
\hline M stage & $\begin{array}{l}\text { Present } \\
\text { Absent }\end{array}$ & $\begin{array}{l}15 \\
12\end{array}$ & $\begin{array}{l}7 \\
5\end{array}$ & 0.034 & 0.872 \\
\hline Survival & $\begin{array}{l}\text { Alive } \\
\text { Died }\end{array}$ & $\begin{array}{l}9 \\
18\end{array}$ & $\begin{array}{l}9 \\
3\end{array}$ & 5.802 & $0.016^{*}$ \\
\hline
\end{tabular}

Diagnostic Performance of PET/CT in the detection of pancreatic cancer:

In this study we found that false positive rate was $22 \%$ while false negative rate was $14 \%$. Sensitivity of PET-SUV in prediction of malignant lesion in the pancreas was $81 \%$, specificity $78 \%$ and accuracy $82 \%$.

Correlation of (SUV) of PET-CT and overall Survival status:

During follow-up of the clinical course of 39 patients included in this study, 21 patients $(58.3 \%)$ were dead while 18 patients $(48.7 \%)$ remained alive with significant difference. $(p=0.001)$.

Mean SUV (>4.5) in malignant pancreatic mass was higher in patients who died compared with patients who still alive $(\leq 4.5)$ with significant difference $(p=0.016)$.

Median OS in patients with SUV (>4.5) were (12.30 \pm 6.50 months) versus (16.60 \pm 6.57 months) in patients with SUV $(\leq 4.5)$ with significant difference $(p=0.010)$. Fig. (1).

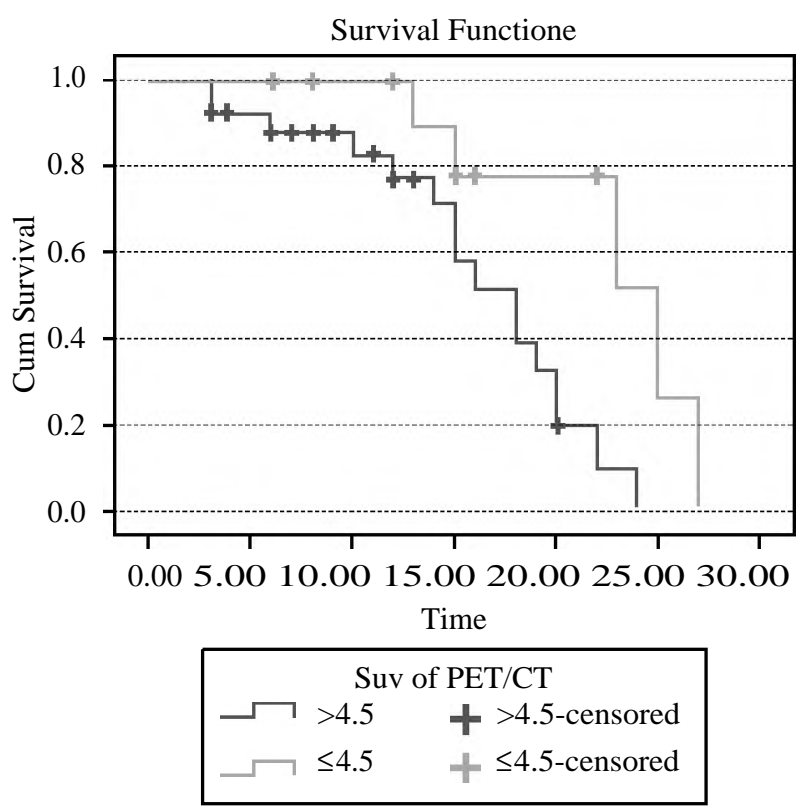

Fig. (1): Kaplan-Meier Analysis: PET/SUV = standard uptake value and Overall survival (OS).

\section{Discussion}

Pancreatic cancer is one of the leading causes of death worldwide [14]. At the time of diagnosis, only $9.7 \%$ of malignant pancreatic lesions are at stage 1 [15]. PET/CT is non-invasive technique plays an important role in staging primary pancreatic tumors and detecting distant metastasis. It is used to detect the metabolism of glucose in malignant lesion which suggested by increased the uptake of $18 \mathrm{~F}-\mathrm{FDG}$ by PET/CT which is presented by SUV [16]. 


\section{Patient presentation:}

Fig. (2): A male patient aged 66 years complained from abdominal pain. The patient was diagnosed to have a psudopancreatic cyst in pancreatic tail by ultrasound. Patient condition deteriorated rapidly with development of ascites and loss of weight. Aspiration revealed malignant ascites and the patient was referred to PET/CT unit for more evaluation. Multi-slice (16) contrast enhanced full dose PET/CT scan of the upper abdomen.
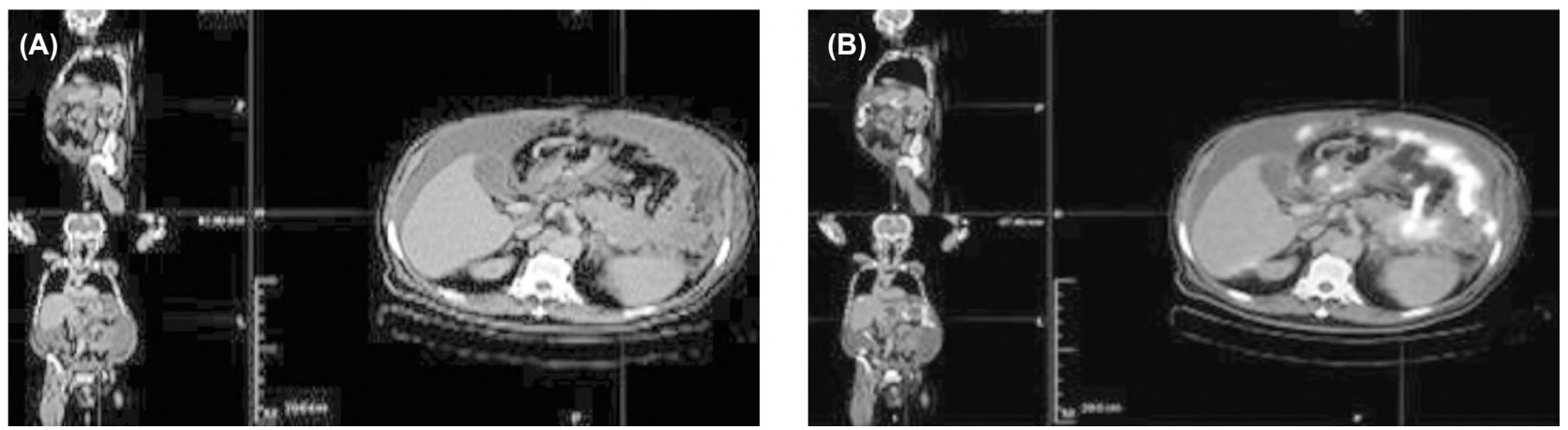

(A\&B): Multiplanar CT \& fusion PET/CT scan showed complex cystic mass in pancreatic tail with focal uptake, SUV: 11.5.
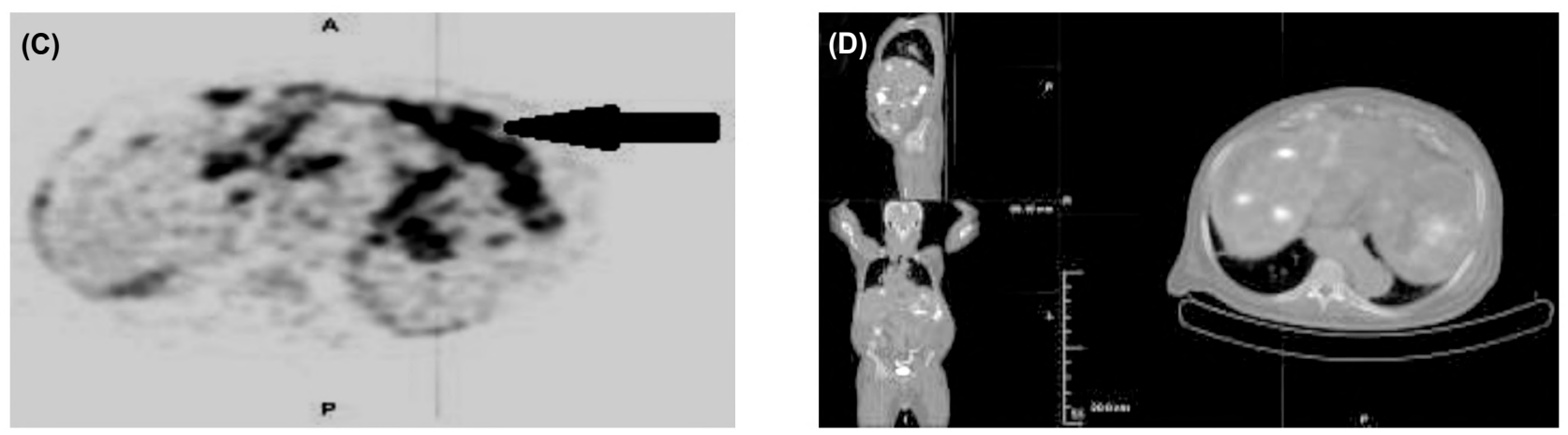

(C\&D): Axial PET/CT scan \& fusion multiplanar PET/CT scan showed abnormal omental uptake (black arrow) and multiple active foci in the liver.

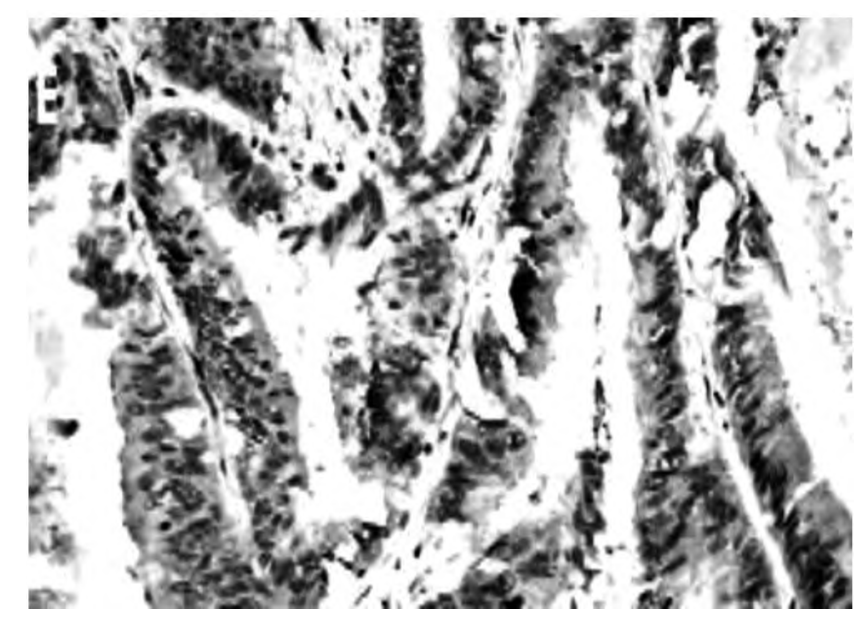

(E): Immunohistochemical analysis showed high expression of ALDH1 positive $[+3]$ indicated well differentiated pancreatic adenocarcinoma [x 400]. 
Fig. (3): A male patient aged 70 years complained from loin pain and jaundice 2 years ago by MDCT. He was diagnosed to have bulky uncinate processes of the pancreas by MDCT. Multi-slice (16) contrast enhanced full dose PET/CT scan of the upper abdomen.
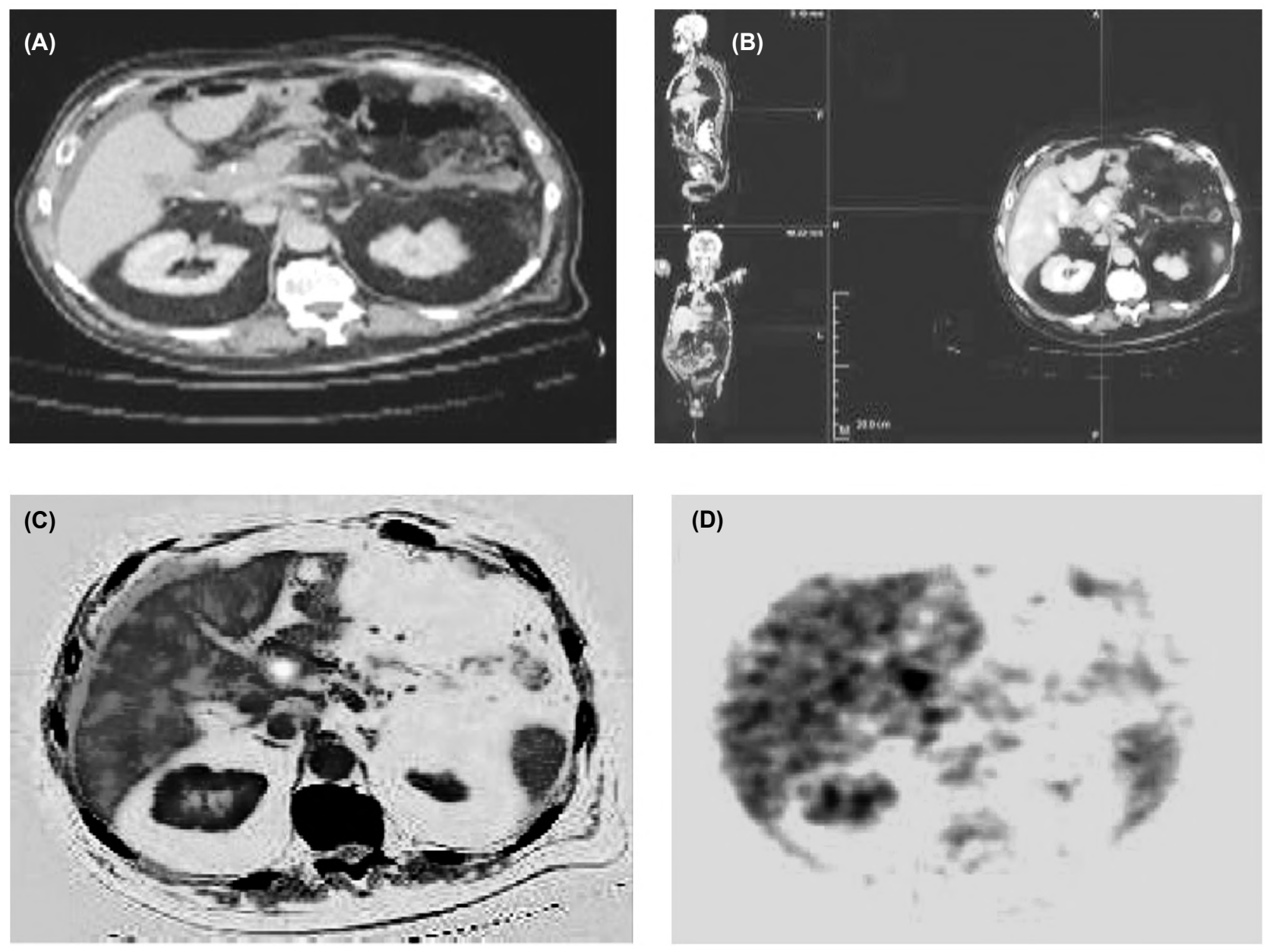

(A,B,C,D): Axial CT \& fusion multiplanar PET/CT scan \& axial PET/CT scan raveled soft tissue mass in the head of pancreas with focal uptake, SUV value : 6.7 .

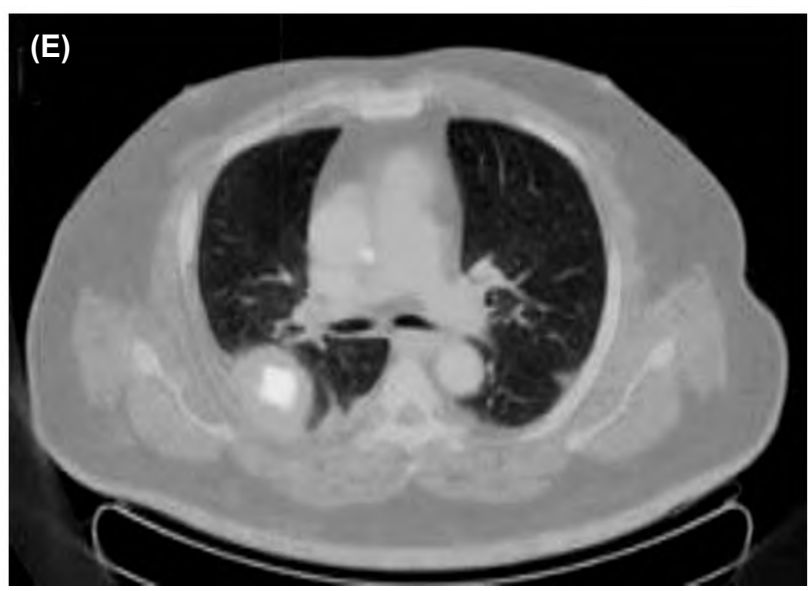

(E): Axial PET /CT scan showed a well-defined right pleural based metastatic pulmonary mass seen in the apical segment of right lower lobe with central metabolic activity, SUV : 10 .

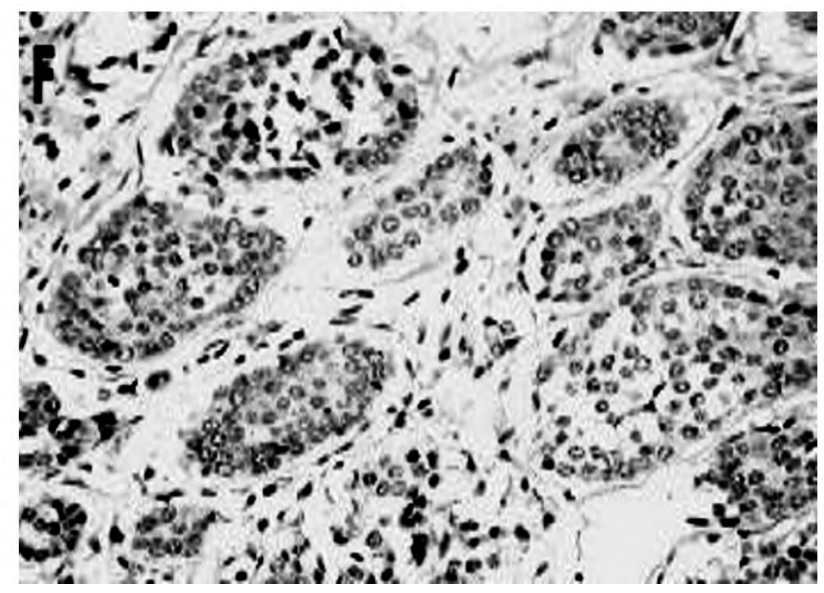

(F): Immunohistochemical analysis showed high expression of ALDH1 positive [+3] indicated low grade pancreatic neuroendocrine tumor $[\mathrm{x} 400]$. 
Fig. (4): A 58-year-old man with progressive weight loss and jaundice. Multi-slice (16) contrast enhanced full dose PET/CT scan of the upper abdomen.
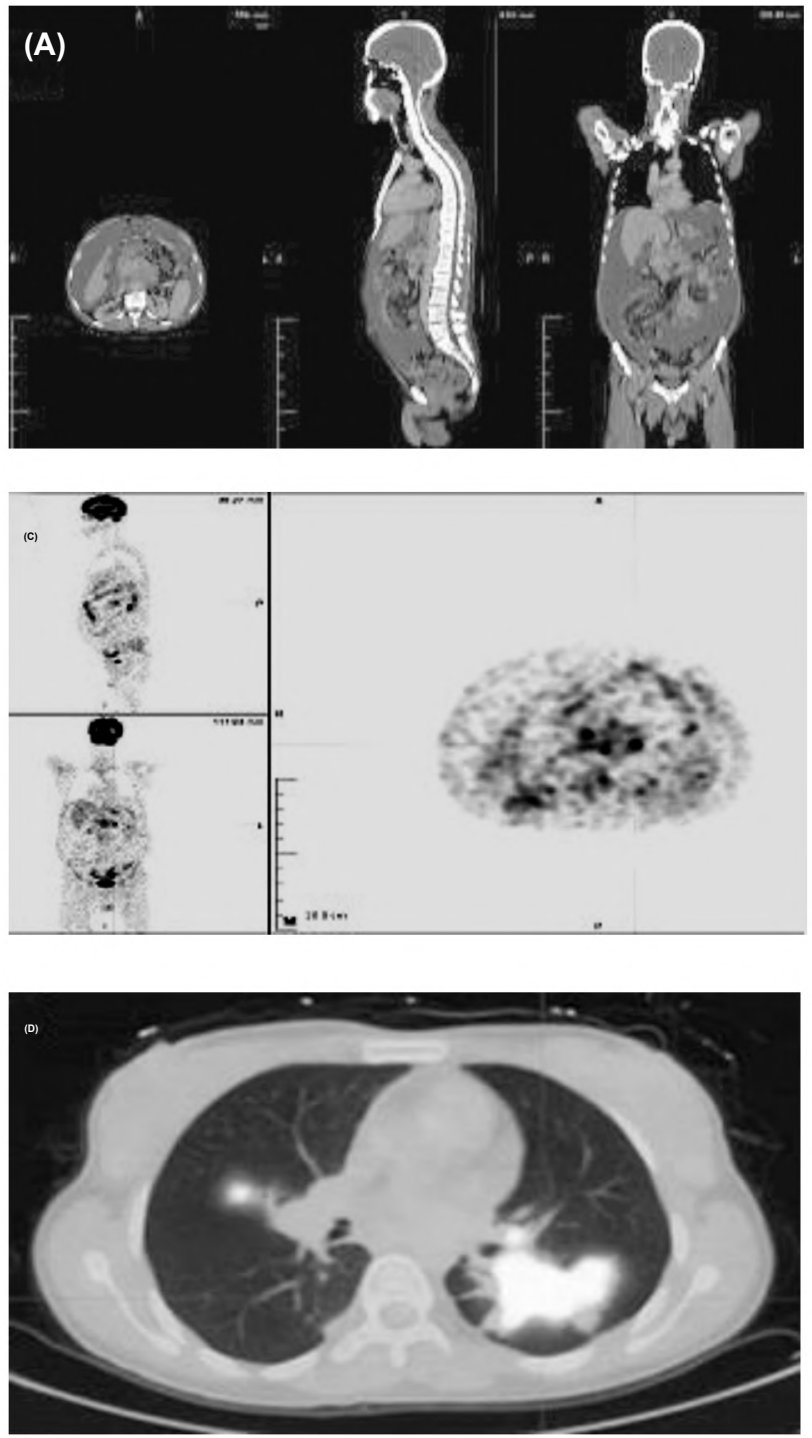

(D): Axial fusion PET/CT showed right hilar lymph node with focal uptake, SUV: 8 with left peri-hilar metastatic pulmonary infiltration extending into apical and anterior segments of left lower lung lobe showing increased metabolic activity, SUV :12.

The study and recognition and of cancer stem cells depend on the presence of antibodies against certain antigens present on cell surface named CD44, CD90, CD24, CD133 and aldehyde dehydrogenase 1 (ALDH1). These markers helped to increase our information about the natural process of pancreatic cancer cells. They make the identification of common features of many types of cancer easier like unrestricted self-renewal ability, specific carcinogenesis or inherent resistance to chemotherapy [17].

Aldehydedehydrogenase 1 (ALDH1) is an important protein secreted and expressed by pancreatic

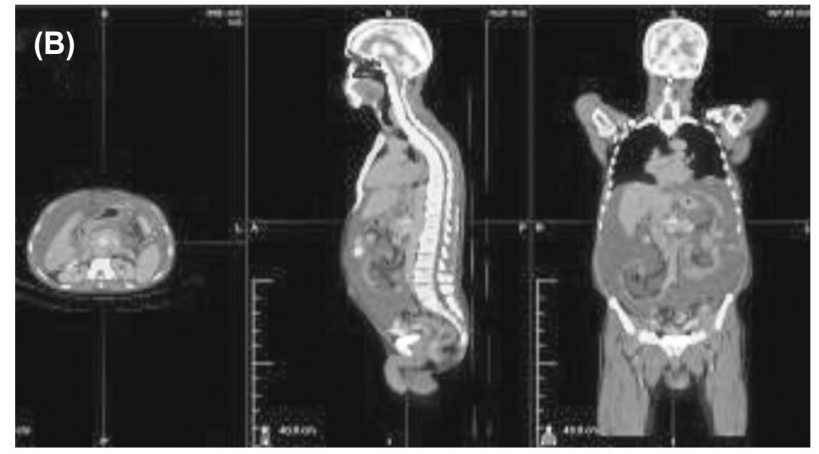

(A,B\},C): Multiplanar CT \& fusion PET/CT scan \& Multiplanar $\mathrm{PET} / \mathrm{CT}$ scan raveled soft tissue mass in the body of pancreas with 2 focal areas of metabolic activity, the most active focus showed SUV : 7.5.

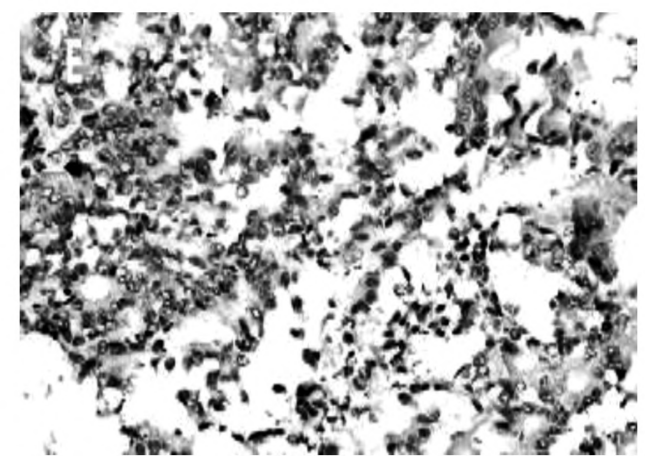

(E): Immunohistochemical analysis showed low expression of ALDH1 negative [+1] indicated poorly differentiated pancreatic adenocarcinoma [x 400].

cancer stem cells. It helps the metabolism of glucose in malignant cells [18]. It produces sufficient amount of extracellular lactate which creates the suitable microenvironment required for the growth, invasion of cancer stem cells and distant metastasis. Poor survival outcomes associated with high expression of ALDH1 [19]. Inhibition the activity of ALDH1 may be helpful in cancer treatment, a few promising number of inhibitors of ALDH1 are discovered [20].

This study included 39 patients suspected of having pancreatic cancer on the basis of MDCT. All patients were studied by PET/CT. We detected 
the expression of ALDH1 as CSC marker immunohistochemically in cancer specimens. The results of PET/SUV were correlated with clincopathological character, ALDH1 and overall survival before therapy.

Before therapy, mean SUV by PET-CT of 4.5 was used as a cut-off value to detect the activity of malignant tumor. Twenty-seven patients (69\%) showed high PET/SUV (more than 4.5) and 12 patients (31\%) had low PET/SUV (less than 4.5).

Many researchers have investigated the prognostic value of PET/CT results in pancreatic cancer patients and they showed absence of standardized cutoff values of SUV to detect the activity of malignant lesion. They concluded that high SUV values of PET/CT scans of pancreatic cancer patients associated with poorer survival outcome [21]

This study showed that SUV values were associated significantly with tumor size in patients with pancreatic cancer $(p=0.027)$ while there were no significant correlation between PET/SUV values and any other clinical or pathological characteristics of included patients.

Similar to our results, the study of Moon et al., [22] which included 21 patients with locally advanced pancreatic cancer, they found that the correlation of the SUV value and tumor size may be due to tumor cellularity.

Sun et al., [23] in their study which included 91 patients diagnosed to have pancreatic cancer by PET/CT before therapy. Using the best cutoff value of $\mathrm{SUV}=5.49$, they divided all patients into 2 groups: High and low SUV groups (SUV >5.49) and (SUV $<5.49$ ). They showed that SUV in patients with cancer pancreas was associated only with tumor size $(p<0.05)$. They concluded in the same study that the clinical and pathological features of all included patients did not correlate with SUV values whether high or low.

False negative rate in this study was $14 \%$. We had 3 patients with false negative results due to low FDG uptake; 1 patient showed small tumor size less than $1 \mathrm{~cm}$, one patient had necrotic tumor and the third one with adenocarcinoma showed desmoplastic reaction surrounded the malignant tumor decreasing FDG uptake. The false positive rate in this study was $22 \%$. We had 4 patients with false positive results due to focal area of FDG activity mainly at pancreatic head and proved to be technical error from FDG activity of nearby bowel in 2 patients. Other 2 patients were due to mass-forming chronic pancreatitis. The diagnostic performance of PET/CT in diagnosis of pancreatic cancer were sensitivity $=81 \%$, specificity $=78 \%$ and accuracy $=82 \%$.

In study by Sun et al., [23], they found that the sensitivity and specificity of SUV of PET/CT in the diagnosis of cancer of pancreas were relatively low $67.5 \%$ and $72.73 \%$ and explained that due to the high value of pre-therapy SUV (5.49). They combined the results of PET/SUV with tumor marker (CA19-9) to improve the diagnostic performance of PET/CT. These indices of PET/CT when combined with CA19-9 increased to $96.25 \%$ and $95.06 \%$.

Zhang et al., [24] in their study suggested that PET/CT have no added advantage in diagnosis of cancer pancreatic patients. They advised that added phase of enhanced PET/CT to CT to obtain single fusion scan may increase the accuracy of PET/CT. Sensitivity of PET in diagnosis, evaluating N staging and in liver metastasis were $91 \%, 64 \%$ and $67 \%$ respectively and the corresponding specificities were $81 \%, 81 \%$ and $96 \%$ respectively.

Some researchers have suggested that the combination of PET/CT with endoscopic ultrasonography is helpful in patients with pancreatic cancer as we add the high sensitivity of PET/CT to the high specificity of endoscopic ultrasonography [25].

Schick et al., [26] in their study which included 27 histopathologically proven pancreatic malignant tumors, found that SUV of malignant lesion was higher than SUV of benign lesions and they stated that sensitivity and specificity of PET/CT were $89 \%$ and $74 \%$ for differentiating between such lesions .They hoped that PET/CT may be able to recognize the mass in chronic pancreatitis differentiate it from malignant lesion. This is may be difficult due to the overlap of SUV values of $\mathrm{PET} / \mathrm{CT}$ of these two lesions.

In this study, $85 \%$ of included patients with high expression of ALDH1 showed SUV more than 4.5. Fifteen percent of patients with low expression of ALDH1 showed PET/SUV more than 4.5. There was statically significant correlation between both parameters $(p=0.001)$. These results suggested that SUV of PET/CT might reflect the expression level of pancreatic cancer stem cells such as ALDH 1.

We found that patients who showed high PETSUV more than 4.5 with presence of high expression of ALDH1 in resected primary malignant mass had a worse median overall survival rate than those 
with a SUV of less than 4.5 with presence of low expression of ALDH1. There was $25 \%$ decrease in the median overall survival (12.30 versus 16.50 months) with statically significant correlation ( $p=$ 0.010 and 0.002 ).

In a study by Rasheed et al. [11], they observed that the presence of ALDH1-positive cells in excised malignant pancreatic tumors was associated with a $22 \%$ decrease in median overall survival compared with ALDH-negative tumors (14 vs 18 months). They concluded that ALDH1 may be a helpful biomarker that estimates long-term clinical course in pancreatic cancer patients but they recommended more large prospective studies to confirm their results.

\section{Conclusion:}

PET-CT is a noninvasive imaging modality that appears to be preoperatively appropriate to cancer pancreatic patients especially in doubtful tumors. Its false positive results in patients with mass forming pancreatitis may lower its specificity. However, PET/CT was a valuable tool to detect lymph nodes invasion and distant metastasis. $\mathrm{PET} / \mathrm{CT}$ was associated with the expression level of cancer stem cells named ALDH1. Increased FDG uptake presented by SUV as well as high expression of ALDH1 in the primary pancreatic malignant tumors were significantly correlated with a worse clinical outcome. So PET/CT-SUV and Cancer stem cells may serve as significant prognostic factors for predicting outcomes in pancreatic cancer patients.

\section{Ethics approval and consent to participate:}

This study was approved by the Institutional Review Board (IRB) and local ethical committee of Tanta university hospital. A written informed consent was received from all patients participate in this study.

\section{References}

1- ZHANG Q., ZENG L., CHEN Y., et al.: Pancreatic Cancer Epidemiology, Detection, and Management. Gastroenterol. Res. Pract, 2016: 8962321, 2016.

2- MUNIRAJ T., JAMIDAR P.A. and ASLANIAN H.R.: Pancreatic cancer: A comprehensive review andupdate. Disease-a-Month., 59 (11): 368-402. doi: 10.1016/j. disamonth.2013.08.001. [PubMed] [CrossRef], 2013.

3- HICKS A.M., CHOU J., CAPANU M., et al.: Pancreas Adenocarcinoma: Ascites, Clinical Manifestations, and Management Implications. Clin. Colorectal Cancer. Dec. 15 (4): 360-368. [PMC free article] [PubMed], 2016.

4- ADAMEK H.E., ALBERT J., BREER H., et al.: Pancreatic cancer detection with magnetic resonance cholangiopancreatography and endoscopic retrograde cholangiopancre- atography: A prospective controlled study. The Lancet, 356 (9225): 190-193. doi: 10.1016/s0140-6736(00)02479x. [PubMed] [CrossRef], 2000.

5- CATALANO C., LAGHI A., FRAIOLI F., et al.: Pancreatic carcinoma: The role of high-resolution multislice spiral $\mathrm{CT}$ in the diagnosis and assessment of resectability. Eur. Radiol., 13: 149-56. [PubMed], 2003.

6- SCHMIDT-HANSEN M., BALDWIN D.R., HASLER E., et al.: PET-CT for assessing mediastinal lymph node involvement in patients with suspected resectable nonsmall cell lung cancer. Cochrane Database Syst. Rev., 11:CD0095192014.

7- NGUYEN P.H., GIRAUD J., CHAMBONNIER L., et al.: Characterization of Biomarkers of Tumorigenic and Chemoresistant Cancer Stem Cells in Human Gastric Carcinoma. Clin. Cancer Res., doi: 10.1158/1078-0432.CCR-152157. [PubMed] [CrossRef], 2016.

8- SIEGEL R.L. and WARD E.M.: "Global cancer incidence and mortality rates and trends-an update," Cancer Epidemiology, Biomarkers and Prevention, Vol. 25, No. 1, pp. 16-27, 2016. View at Publisher o View at Google Scholar o View at Scopus.

9- RAO C.V. and MOHAMMED ALTAF: New insights into pancreatic cancer stem cells. World J. Stem Cells, 7 (3): 547-555, 2015.

10- ZHANG Y., YANG C.H., CHENG H.E., et al.: Novel agents for pancreatic ductal adenocarcinoma: Emerging therapeutics and future directions. Journal of Hematology \& Oncology, 11:14, 2018.

11- RASHEED Z.A., YANG J., WANG Q., et al.: Prognostic significance of tumorigenic cells with mesenchyma features in pancreatic adenocarcinoma. J. Natl. Cancer Inst., 102: 340-351, 2010.

12- KAUHANEN S., RINTA-KIIKKA I., KEMPPAINEN J., et al.: Accuracy of 18F-FDG PET/CT, Multidetector CT, and MR Imaging in the Diagnosis of Pancreatic Cysts: A Prospective Single-Center Study. J. Nucl. Med. Aug., 56 (8): 1163-8, 2015.

13- ONUL A.B., COLVARD M.D., PARADISE W.A., et al.: Application of Immunohistochemical Staining to Detect Antigen Destruction as a Measure of Tissue Damage. J. Histochem Cytochem. Sep., 60(9): 683-693. doi: $10.1369 / 0022155412452146,2012$

14- American Cancer Society. [cited March 12, 2018]. In: Cancer Statistics Center [Internet]. Available from: https://cancerstatisticscenter.cancer.org ., 2018.

15- National Cancer Institute. [cited March 14, 2018]. In: Pancreatic Cancer Cancer Stat Facts [Internet]. Available from: https://seer.cancer.gov/., 2018.

16- CASNEUF V., DELRUE L., KELLES A., VAN DAMME N., VAN HUYSSE J., BERREVOET F., et al.: Is combined 18F-fluorodeoxyglucose-positronemission tomography/ computed tomography superior to positronemission tomography or computed tomography alone for diagnosis,staging and restaging of pancreatic lesions? Acta. Gastroenterol. Belg., 70: 331-8. [PMID 18330088], 2007.

17- NGUYEN P.H., GIRAUD J., CHAMBONNIER L., et al. Characterization of Biomarkers of Tumorigenic and Chemoresistant Cancer Stem Cells in Human Gastric Carcinoma. 
Clin. Cancer Res. doi: 10.1158/1078-0432.CCR-152157.[PubMed] [CrossRef], 2016.

18-RANI R. and KUMAR V.: Recent update on human lactate dehydrogenase enzyme 5 (hLDH5) inhibitors: A promising approach for cancer chemotherapy. J. Med. Chem., 59: 487-96. [PubMed], 2016.

19- BRANDI J., DANDO I., POZZA E.D., et al.: Proteomic analysis of pancreatic cancer stem cells: Functional role of fatty acid synthesis and mevalonate pathways. J. Proteomics., 150: 310-322, 2017.

20- ALTAMIMI A.S., ALAFEEFY A.M., BALODE A., et al.: Symmetric molecules with 1,4-triazole moieties as potent inhibitors of tumour-associated lactate dehydrogenase-A. J. Enzyme Inhib Med. Chem., 33: 147-150, 2018.

21- CHOI H.J., KANG C.M., LEE W.J., et al.: Prognostic value of $18 \mathrm{~F}$-fluorodeoxyglucose positron emission tomography in patients with resectable pancreatic cancer. Yonsei Med. J., 54: 1377-1383. [PMC free article] [PubMed], 2013.

22- MOON S.Y., JOO K.R., SO Y.R., et al.: Predictive value of maximum standardized uptake value (SUVmax) on 18F-FDG PET/CT in patients with locally advanced or metastatic pancreatic cancer. Clin. Nucl. Med., 38: 778783. [PubMed], 2013.
23- SUN Y., DUAN Q., WANG S., et al.: Diagnosis of pancreatic cancer using 18F-FDG PET/CT and CA19-9 with SUVmax association to clinical characteristics. JBUON, 20 (2): 452-459, 2015.

24- ZHANG L., SANAGAPALLI S. and STOITA A.: Challenges in diagnosis of pancreatic cancer: World J. Gastroenterol. May 21, 24 (19): 2047-2060, 2018.

25- TANG S., HUANG G., LIU J., LIU T., et al : Usefulness of 18F-FDG PET, combined FDG-PET/CT and EUS in diagnosing primary pancreatic carcinoma: A meta-analysis. Eur. J. Radiol., 78:142-150. [PubMed] [DOI], 2011.

26- SCHICK V., FRANZIUS C., BEYNA T., et al.: Diagnostic impact of 18F-FDG PET-CT evaluating solid pancreatic lesions versus endosonography, endoscopic retrograde cholangio-pancreatography with intraductal ultrasonography and abdominal ultrasound. Eur. J. Nucl. Med. Mol. Imaging, 35 (10): 1775-1785. Crossref, Medline, Google Scholar, 2008.

27- KATO K., NIHASHI T., IKEDA M., et al.: Limited efficacy of (18)F-FDG PET/CT for differentiation between metastasis-free pancreatic cancer and mass-forming pancreatitis. Clin. Nucl. Med., 38: 417-421. [PubMed] [DOI], 2013.

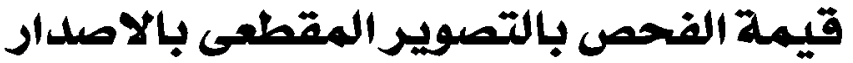

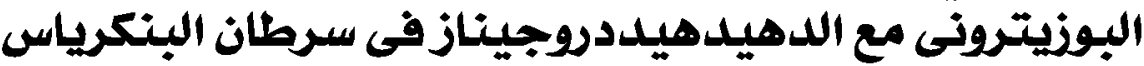

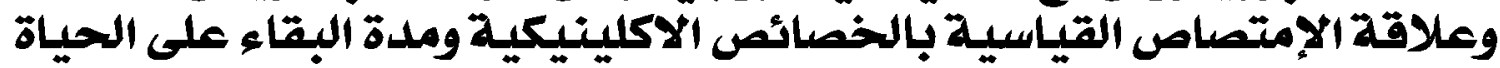

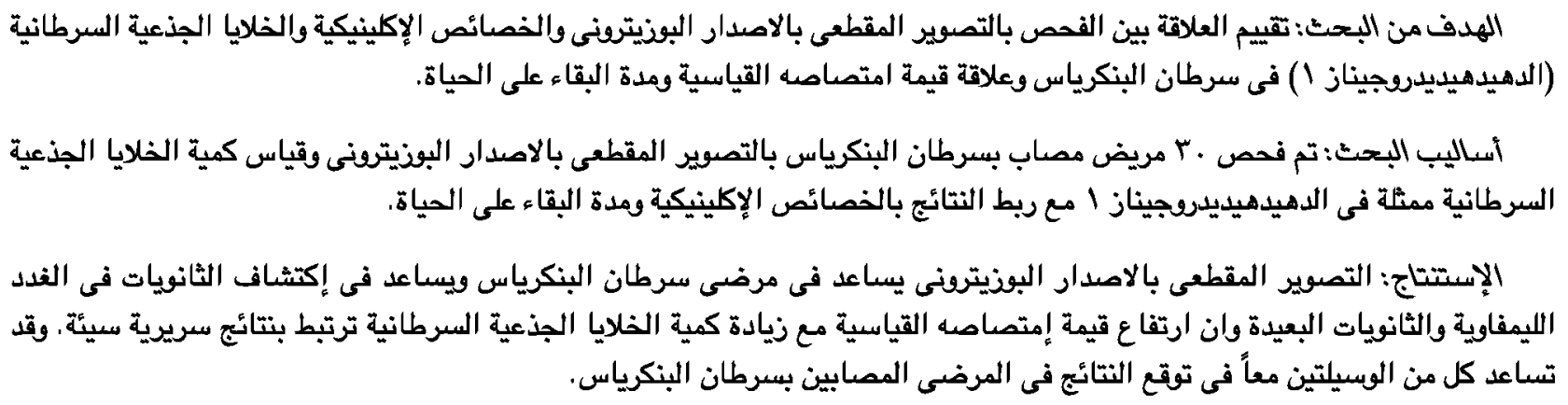

